\title{
Interação medicamentosa e a farmacoterapia de pacientes geriátricos com síndromes demenciais
}

\author{
Drug interaction and pharmacotherapy of geriatric patients with dementia
}

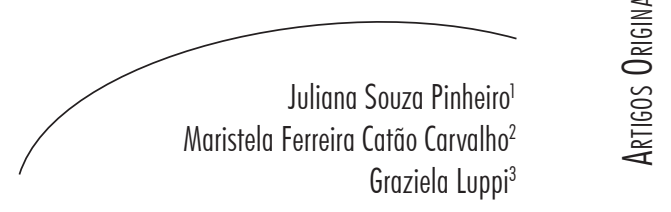

\section{Resumo}

Objetivo: Investigar as possíveis interações medicamentosas, a fim de identificar os prováveis eventos adversos relacionados à farmacoterapia das síndromes demenciais. Metodologia: A amostra foi constituída por 48 idosos com idade igual ou superior a 60 anos, portadores de síndromes demenciais atendidos no grupo terapêutico da equipe multiprofissional do Instituto Paulista de Geriatria e Gerontologia (IPGG). A primeira etapa foi a descrição da farmacoterapia geriátrica dos pacientes com síndromes demenciais. A segunda foi a análise estatística dos dados coletados com base no pacote Stata ${ }^{\circledR} 9.0$, adotando-se $p<0,001$, e a análise das interações medicamentosas foi baseada na utilização do programa Lexi-Interact ${ }^{\mathrm{TM}}$. Resultados: Dos pacientes avaliados, verificouse predomínio de pacientes do gênero feminino (66,7\%). Em ambos os gêneros, a demência de Alzheimer foi o diagnóstico de maior prevalência, sendo $60 \%$ nas mulheres e $80 \%$ nos homens. Também se verificou maior adesão nos pacientes com diagnóstico de Alzheimer (81,8\%). No que diz respeito aos medicamentos utilizados, verificaramse 41 diferentes fármacos prescritos. Entre eles, os mais usados foram os do sistema cardiovascular (30,8\%), seguidos dos medicamentos que atuam no nível central (29,8\%). A polifarmácia foi verificada em $73,3 \%$ dos pacientes, mais predominante em pacientes do sexo feminino (54,5\%), portadoras da demência de Alzheimer (72,7\%), com grau moderado (50\%). Entre as interações medicamentosas, destacaram-se as decorrentes do uso concomitante de medicamentos anti-hipertensivos (30,5\%), dentre os quais os inibidores da enzima conversora de angiotensina (IECA) e os diuréticos (2,2\%). Conclusão: A interação medicamentosa, como evento precursor do uso de medicamentos, pode resultar em efeitos potencialmente perigosos, como visto nas interações medicamentosas mediadas no uso de medicamentos hipotensores, cujo evento tem relação indireta com o grau de demência, e consequentemente comprometendo a evolução clínica dos pacientes diagnosticados como portadores de síndromes demenciais.

\footnotetext{
Programa de Aprimoramento Multiprofissional em Gerontologia. Instituto Paulista de Geriatria e Gerontologia. São Paulo, SP, Brasil.

2 Diretoria Técnica de Serviços de Saúde, Núcleo de Assistência e Atenção Farmacêutica. Instituto Paulista de Geriatria e Gerontologia. São Paulo, SP, Brasil.

3 Núcleo de Assistência e Atenção Farmacêutica. Instituto Paulista de Geriatria e Gerontologia. São Paulo, SP, Brasil.
}

Palavras-chave: Interações de Medicamentos. Inibidores da Colinesterase. Uso de Medicamentos. Saúde do Idoso. 


\section{Abstract}

Objective: To investigate possible drug interactions to identify the probable adverse events related to the pharmacotherapy of dementia. Methods: The sample consisted of 48 elderly aged over 60 years, patients with dementia treated in the therapeutic group of multidisciplinary team from the Instituto Paulista de Geriatria e Gerontologia (IPGG). The first step was the description of geriatric pharmacotherapy of patients with dementia. The second was the statistical analysis of data collected based on the package Stata ${ }^{\circledR} 9.0$, adopting $p<0.001$; the analysis of drug interactions was based on the use of the Lexi-Interact ${ }^{\mathrm{TM}}$ program. Results: Among the patients, there was predominance of female patients (66.7\%). In both genders, Alzheimer's disease was the most prevalent diagnosis, and 60\% in women and $80 \%$ men. It was also found higher adherence in patients diagnosed with Alzheimer's (81.8\%). With respect to drugs used, it was found 41 different prescribed drugs. Among them, the most used were the cardiovascular system $(30.8 \%)$, followed by drugs that act centrally $(29.8 \%)$. Polypharmacy was observed in $73.3 \%$ of patients, more prevalent in female patients $(54.5 \%)$, living with Alzheimer's dementia (72.7\%), moderate (50\%). Among drug interactions, those arising form concomitant use of anti-hypertensive drugs (30.5\%) stand out, including angiotensin-converting enzyme (ACE) inhibitors and diuretics (2.2\%). Conclusion: Drug interaction, as precursor event of medication can result in potentially dangerous effects, as seen in drug interactions mediated in the use of hypotensive drugs, whose event has indirect relationship with the degree of dementia and consequently jeopardizes progress treatment of patients diagnosed as having dementia.
Key words: Drug

Interactions. Cholinesterase Inhibitors. Drug Utilization. Health of the Elderly.

\section{INTRODUÇÃO}

O processo de envelhecimento populacional vem ocorrendo rapidamente nos países em desenvolvimento, o que desencadeia a necessidade de recursos de saúde, em grande parte devido à alta prevalência de doenças crônicodegenerativas, sendo estas as principais causas de tratamentos complexos e prolongados que geram maior custo nas internações, equipamentos e medicamentos. ${ }^{1,2}$

Estima-se que entre 1950 e 2025 a população idosa no Brasil crescerá 16 vezes, contra cinco vezes a população mundial. A maior preocupação serão as doenças crônico-degenerativas, dentre as quais as síndromes demenciais. Em 2003, no município de São Paulo, verificou-se prevalência de deterioração cognitiva de $3,4 \% .{ }^{3}$ O diagnóstico para as síndromes demenciais, que se caracterizam pela perda progressiva das funções cognitivas, é realizado com base em aspectos clínicos classificados como leve, moderado e grave. ${ }^{1}$
Os estudos populacionais sobre a prevalência de demência nos países desenvolvidos e no Brasil são escassos, podendo-se considerar a demência de Alzheimer (DA) a mais prevalente das demências. No Estado de São Paulo a demência de Alzheimer é a principal causa de deterioração cognitiva, chegando a $62,8 \%$, enquanto que a demência vascular representa $8,8 \%{ }^{1}$

Apesar da inexistência de estudos específicos envolvendo idosos com demência, alguns autores descrevem mudanças que alteram o perfil farmacológico, como distribuição de líquido corpóreo, massa muscular e gordura corpórea, que são parâmetros responsáveis por alterações que afetam o metabolismo e eliminação dos medicamentos utilizados por esses idosos, tornando-os ainda mais susceptíveis em relação aos idosos não demenciados. ${ }^{1,4-6}$

O perfil farmacológico do tratamento das síndromes demenciais é responsável pela maior susceptibilidade às interações medicamentosas, uma vez que esse pode afetar sensivelmente tanto 
a farmacocinética como a farmacodinâmica da maioria dos fármacos, comprometendo a duração da atividade farmacológica, ou ainda aumentando a toxicidade medicamentosa e o risco de reações adversas. ${ }^{1,7}$

As interações medicamentosas têm sido um importante objeto de investigação, uma vez que o aumento das doenças crônico-degenerativas em pacientes idosos implica aumento do consumo de medicamentos, havendo por consequência a polifarmácia e a iatrogenia. ${ }^{4}$ Os idosos com demência, todavia, estão mais susceptíveis à polifarmácia devido à idade avançada, presença de outras condições patológicas, dependência funcional e fragilidade. ${ }^{1,4,5}$

O seguimento farmacoterapêutico (SFT), no entanto, é um método clínico considerado um componente da atenção farmacêutica (AF), utilizado como instrumento de investigação, no qual o farmacêutico se responsabiliza pelas necessidades do usuário relacionadas ao medicamento, detectando, prevenindo e resolvendo problemas relacionados aos medicamentos (PRM) de forma sistemática, contínua e documentada, com o objetivo de alcançar resultados definidos, buscando melhoria da qualidade de vida do usuário. ${ }^{8}$

Em 2007, na Espanha, o grupo de Investigação em Atenção Farmacêutica da Universidade de Granada propôs a implementação do seguimento farmacoterapêutico, conhecido como metodologia Dáder, baseado na obtenção de informações sobre a farmacoterapia do paciente, problemas de saúde e medicamentos administrados, com o objetivo de atingir resultados concretos que melhorassem a qualidade de vida de seus doentes.

Este estudo tem como objetivo investigar as possíveis interações medicamentosas, a fim de identificar os prováveis eventos adversos relacionados à farmacoterapia das síndromes demenciais, bem como descrever o perfil dos idosos portadores de síndromes demenciais referente às características sociodemográficas e caracterizar a farmacoterapia quanto ao uso de medicamentos e polifarmácia.

\section{METODOLOGIA}

Trata-se de estudo descritivo exploratório de campo, desenvolvido em duas etapas, sendo a primeira a descrição da farmacoterapia geriátrica dos pacientes com síndromes demenciais. Esse dado foi colhido frente aos relatos do cuidador e/ou responsáveis pelos pacientes e pelas informações descritas no prontuário, frente ao diagnóstico estabelecido.

A segunda etapa foi exploratória, baseada na análise dos dados colhidos e na identificação das supostas interações medicamentosas. Este estudo foi realizado em uma instituição pública de serviço a saúde do Estado de São Paulo, nomeada como IPGG (Instituto Paulista de Geriatria e Gerontologia José Ermírio de Moraes), no período de março de 2010 a fevereiro de 2011.

\section{População e amostra}

A amostra foi constituída por 48 idosos com idade igual ou superior a 60 anos, diagnosticados como portadores de síndromes demenciais. Esses pacientes pertencem a um grupo terapêutico da equipe multiprofissional do IPGG. A amostra foi homogeneizada de acordo com os critérios de inclusão e exclusão. O critério de inclusão se referiu a pacientes com diagnóstico de demência há pelo menos três meses. Os critérios de exclusão foram: paciente com diagnóstico de demência classificado como grau grave ou com provável síndrome demencial; pacientes que não apresentaram a classificação do teste Clinical Dementia Rating (CDR) de pelo menos seis meses; e pacientes cujos cuidadores e/ou responsáveis não assinaram o Termo de Consentimento Livre e Esclarecido.

\section{Coleta de dados}

Contatos telefônicos foram realizados para convidar os cuidadores e/ou responsáveis pelos idosos com síndromes demenciais a participar da pesquisa. Os encontros foram agendados de acordo com a disponibilidade dos mesmos. 
A coleta dos dados primeiramente se realizou com base nas informações obtidas em prontuário, obedecendo aos critérios de inclusão e exclusão. As informações se referiram ao teste de CDR, à conduta médica frente ao diagnóstico individual do paciente e ao monitoramento do tratamento farmacológico. Após, aplicou-se um formulário com perguntas abertas e fechadas, baseado em modelos de documentos usados na metodologia Dáder $^{10}$ e no Protocolo Clínico de Diretrizes Terapêuticas ao Tratamento do Alzheimer. ${ }^{11}$

\section{Variáveis de estudo}

A variável dependente deste estudo é o uso de medicamento. Para a obtenção dos dados sobre o uso de medicamento, coletou-se primeiramente informação no prontuário e, em seguida, verificou-se junto ao cuidador os medicamentos em uso com as seguintes perguntas: 1) "Mostreme as receitas do paciente?"; 2) "Mostre-me os remédios do paciente?"; 3) "Está usando outro medicamento que não está prescrito?". Para a classificação dos medicamentos, foi utilizado o sistema de classificação de acordo com AnatomicalTherapeutical-Chemical Classification System (ATC). ${ }^{12}$ A polifarmácia foi definida como o uso de cinco ou mais medicamentos. ${ }^{11,13}$

As síndromes demenciais foram classificadas como demência de Alzheimer, demência vascular e demência mista; e a intensidade das demências, em grau leve, moderada e grave. A fim de diferenciar as demências mista e vascular, foram verificadas as doenças associadas.

A caracterização sociodemográfica dos sujeitos da pesquisa foi realizada por meio da análise das variáveis: gênero, idade e escolaridade. A não adesão ao tratamento medicamentoso foi considerada como a situação em que o cuidador de idosos demenciados não seguia o tratamento farmacológico prescrito.

\section{Análise dos dados}

As análises estatísticas dos dados foram realizadas utilizando-se o programa Stata ${ }^{\circledR}$
9.0. Foi realizada análise bivariada por meio do teste do qui-quadrado de Pearson, tendo como variáveis: gênero, idade, escolaridade, tipo de cuidador, doenças associadas, etilismo e tabagismo; adotou-se nível de significância de $p<0,001$. As interações medicamentosas foram analisadas a partir de princípios ativos isolados, por meio do programa Lexi-Interact ${ }^{\mathbf{T M}}$; foram excluídos da análise os medicamentos de uso tópico, devido a sua baixa absorção. Também foi realizado o levantamento bibliográfico nas bases de dados da Biblioteca Virtual de Saúde BIREME, SciELO, PubMed, no período de março a dezembro de 2010.

\section{Pressupostos éticos}

Este estudo foi realizado após a aprovação pelo Comitê de Ética em Pesquisa do Instituto de Saúde, registrado sob o n ${ }^{\circ}$ 023/2010, na cidade de São Paulo. Todos os idosos entrevistados foram acompanhados de um cuidador e/ou responsável, o qual assinou o Termo de Consentimento Livre e Esclarecido, respeitando-se, entre outros, o anonimato dos sujeitos da pesquisa, tal qual estabelecido na autorização institucional para a realização da investigação. Todos puderam obter acesso a informações sobre procedimentos, riscos e benefícios relacionados à pesquisa, inclusive para esclarecer eventuais dúvidas.

\section{RESULTADOS}

\section{Perfil da população estudada}

Dos 48 idosos pertencentes à amostra inicial, foram excluídos: $54,2 \%$ (26), por serem classificados como grau de demência grave; $4,5 \%$ (2), por óbito e $10,4 \%$ (5), por recusa dos cuidadores e/ou responsáveis em participar da pesquisa.

No que diz respeito às características clínicodemográficas dos 15 pacientes avaliados, cuja idade média foi 80 anos, verificou-se predomínio de pacientes do gênero feminino (66,7\%), com maior prevalência no diagnóstico de demência de Alzheimer (72,7\%). Em relação à idade, a 
faixa etária mais prevalente foi a de 75 a 79 anos $(33,3 \%)$, dos quais $50 \%$ se referiram à demência mista e 36,3\% à demência de Alzheimer, conforme descrito na tabela 1 .

Tabela 1- Distribuição das características clínico-demográficas, segundo a classificação das síndromes demenciais. São Paulo-SP, 2010-2011.

\begin{tabular}{|c|c|c|c|c|c|}
\hline \multirow[t]{2}{*}{ Variáveis } & \multicolumn{4}{|c|}{ Síndromes Demenciais } & \multirow[b]{2}{*}{$\mathrm{p}$} \\
\hline & $\begin{array}{l}\text { Demência de } \\
\text { Alzheimer }\end{array}$ & $\begin{array}{l}\text { Demência } \\
\text { Vascular }\end{array}$ & $\begin{array}{l}\text { Demência } \\
\text { Mista }\end{array}$ & Total & \\
\hline Sexo & & & & & 0,711 \\
\hline Masculino & 27,3 & 50 & 50 & 33,3 & \\
\hline Feminino & 72,7 & 50 & 50 & 66,7 & \\
\hline TOTAL & 100 & 100 & 100 & 100 & \\
\hline Idade & & & & & 0,249 \\
\hline $65-69$ & 9,1 & 0 & 0 & 6,7 & \\
\hline $70-74$ & 9,1 & 50 & 0 & 13,3 & \\
\hline $75-79$ & 36,3 & 0 & 50 & 33,3 & \\
\hline $80-84$ & 27,3 & 0 & 0 & 20 & \\
\hline $90-94$ & 18,2 & 50 & 0 & 20 & \\
\hline $95-99$ & 0 & 0 & 50 & 6,7 & \\
\hline TOTAL & 100 & 100 & 100 & 100 & \\
\hline Grau demência & & & & & 0,362 \\
\hline Leve & 54,5 & 0 & 50 & 46,7 & \\
\hline Moderada & 45,5 & 100 & 50 & 53,3 & \\
\hline TOTAL & 100 & 100 & 100 & 100 & \\
\hline Adesão & & & & & 0,468 \\
\hline $\operatorname{Sim}$ & 69,3 & 55,6 & 53,8 & 66 & \\
\hline Não & 30,7 & 44,4 & 46,2 & 35 & \\
\hline TOTAL & 100 & 100 & 100 & 100 & \\
\hline
\end{tabular}

A maior prevalência de adesão ao tratamento medicamentoso se referiu ao moderado (53,3\%), frente ao diagnóstico de demência de Alzheimer $(62,5 \%)$, seguido da demência vascular $(25 \%)$, enquanto que o grau de demência leve também se verificou maior prevalência na demência de Alzheimer (85,7\%), seguindo da demência mista $(14,3 \%)$. A maioria desses idosos dependia de cuidadores que assumiam a responsabilidade do tratamento medicamentoso e/ou implementação do tratamento não farmacológico. Vale ressaltar que $93,3 \%$ dos cuidadores eram do gênero feminino, apresentando idade média de 56 anos, sendo eles, em sua totalidade, $80 \%$ de cuidadores informais e $20 \%$ de formais.

Dentre as justificativas feitas em relação à não adesão ao tratamento prescrito, ressaltamse: falta de disponibilidade do cuidador em estar junto com o idoso no horário de administrar os 
medicamentos; falta de orientação quanto ao uso dos medicamentos prescritos ou ainda porque o paciente se nega a tomar os medicamentos.

Perfil da terapia farmacológica

No que diz respeito aos medicamentos utilizados, verificaram-se 41 diferentes fármacos prescritos. Os mais usados foram os prescritos para o sistema cardiovascular (30,8\%), sendo os mais comuns os inibidores da enzima conversora de angiotensina (IECA) (4,7\%) e os diuréticos tiazídicos $(3,8 \%)$. A segunda classe mais utilizada foram os medicamentos que atuam a nível central $(29,8 \%)$, sendo os mais frequentes os inibidores da acetilcolinesterase (IACh) (13,9\%) e os analgésicos (3,8\%). Os medicamentos de uso dermatológico $(0,9 \%)$ apresentaram baixa prevalência, conforme o descrito na tabela 2 .

Tabela 2- Medicamentos utilizados pelos idosos, segundo o primeiro e segundo níveis da Anatomical Therapentic Chemical Classification (ATC).São Paulo-SP, 2010-2011.

\begin{tabular}{|c|c|c|}
\hline Classificação ATC & & $\%$ \\
\hline Sistema Cardiovascular (C) & & 31,15 \\
\hline Enalapril & (C09AA02) & 4,72 \\
\hline Hidroclorotiazida & (C03AA03) & 3,78 \\
\hline Anlodipina & (C08CA01) & 2,83 \\
\hline Atenolol & (C07AB03) & 2,83 \\
\hline Atorvastatina & (C10AA05) & 2,83 \\
\hline Losartan & (C09CA01) & 2,83 \\
\hline Sinvastatina & (C10AA01) & 2,83 \\
\hline Captopril & (C09AA01) & 1,90 \\
\hline Ciprofibrato & (C10AB08) & 1,90 \\
\hline Ezetimiba & (C10BA02) & 0,94 \\
\hline Carvedilol & (C07AG02) & 0,94 \\
\hline Espirolactona & (C03DA01) & 0,94 \\
\hline Furosemida & (C03CA01) & 0,94 \\
\hline Troxerrutina & (C05CA54) & 0,94 \\
\hline Sistema nervoso central $(\mathrm{N})$ & & 29,26 \\
\hline Donepezil & (N06DA02) & 13,21 \\
\hline Paracetamol & (N02BE01) & 3,78 \\
\hline Setralina & (N06AB06) & 3,78 \\
\hline Quetiapina & (N05AH04) & 2,83 \\
\hline Rivastigmina & (N06DA03) & 1,90 \\
\hline Diazepan & (N05BA01) & 0,94 \\
\hline Dipirona & (N02BB02) & 0,94 \\
\hline Citalopram & (N06AB04) & 0,94 \\
\hline Galantamina & (N06DA04) & 0,94 \\
\hline
\end{tabular}




\begin{tabular}{lcc}
\hline \multicolumn{1}{c}{ Classificação ATC } & $\%$ \\
\hline Aparelho digestivo e metabolismo (A) & & 18,86 \\
Omeprazol & (A02BC01) & 11,32 \\
Complexo B & (A11EA) & 1,89 \\
Metformina & (A10BA02) & 1,89 \\
Carbonato de cálcio & (A11AA02) & 0,94 \\
Dimenidrato & (A11HA02) & 0,94 \\
Glibenclamida & (A10BB01) & 0,94 \\
Insulina & (A10AE01) & 0,94 \\
Sangue e órgãos hematopoiéticos (B) & & 12,26 \\
Ácido acetilsalić́lico & (B01AC06) & 9,43 \\
Ácido Fólico & (B03BB01) & 1,89 \\
Cumarina & (B01AA03) & 0,94 \\
Sistema músculo esquelético (M) & & 2,82 \\
Nimesulida & $($ M01AX17) & 0,94 \\
Risedronato & (M05BA07) & 0,94 \\
Alopurinol & (M04AA01) & 0,94 \\
Órgãos dos sentidos (S) & & 2,82 \\
Brimonidina & (S01EA05) & 0,94 \\
Timolol & (S01ED01) & 0,94 \\
Travoprosta & (S01EE04) & 0,94 \\
Hormônios de uso sistêmico, excluindo hormônios sexuais (H) & 1,89 \\
Levotiroxina sódica & & 1,89 \\
Dermatológico (D) & 0,94 \\
Dexametasona & & 0,94 \\
\hline TOTAL & & 100,0 \\
\hline & & \\
\hline & & \\
\hline
\end{tabular}

A polifarmácia foi verificada em $73,3 \%$ dos pacientes, mais predominante em pacientes do sexo feminino $(54,5 \%)$ com 70 anos ou mais $(71,4 \%)$, portadoras da demência de Alzheimer (72,7\%), com o grau moderado (50\%).

\section{Perfil das interações medicamentosas}

Ao avaliar a farmacoterapia prescrita, foram identificadas 46 possíveis interações medicamentosas. Somente foram identificadas interações farmacocinéticas, sendo estas responsáveis, principalmente, pelas alterações no metabolismo dos princípios ativos envolvidos. Não foram encontrados na literatura ${ }^{1,4,6,7,10}$ relatos envolvendo interações entre os medicamentos prescritos: ciprofibrato, nimesulida, dipirona, cumarina e troxerrutina.

A partir do pressuposto, verificou-se que as interações medicamentosas mais frequentes acontecem entre os anti-hipertensivos (30,5\%), seguida das decorrentes do uso concomitante de beta bloqueadores einibidores da acetilcolinesterase (IACh) (8,8\%). Enquanto que as de menor 
frequência acontecem entre os inibidores da enzima conversora de angiotensina (IECA) e os diuréticos $(2,2 \%)$, conforme o referido na tabela 3. Os princípios ativos estão descritos no quadro 1.

Quadro 1- Tipos de interações medicamentosas potenciais com os respectivos princípios ativos e efeitos envolvidos.

Interação medicamentosa potencial: Hipotensor - Hipotensor

Princípios ativos: Atenolol/Losartan, Atenolol/Enalapril, Brimonidina/Timolol, Anlodipina/Hidroclorotiazida, Anlodipina/ Losartan, Carvedilol/Furosemida, Carvedilol/Hidroclorotiazida, Furosemida/Hidroclorotiazida, Furosemida/Losartan,

Furosemida/Espirolactona,Hidroclorotiazida/Losartan,

Hidroclorotizida/Anlodipina

Efeito: Aumento do efeito hipotensor

Interação medicamentosa potencial: CYP3A4 substrato Inibidores da isoenzima CYP3A4

Princípios ativos: Timolol/Donepezil, Atenolol/Donepezil Atenolol/Rivastigmina, Carvedilol/Donepezil

Efeito: Redução do metabolismo dos substratos desta via

Interação medicamentosa potencial: IECA - Diuréticos tiazídicos

Princípios ativos: Captopril/ Hidroclorotiazida

Enalapril/ Hidroclorotiazida

Efeito: Desenvolvimento de hipotensão postural aguda

Interação medicamentosa potencial: Carvedilol - Inibidores isoenzima CYP2C9

Princípios ativos: Carvedilol/Omeprazol, Carvedilol/Losartan Efeito: Redução do metabolismo do carvedilol

Interação medicamentosa potencial: Salicilato - Diurético de alça

Princípios ativos: Ácido acetilsalicilico/Furosemida

Efeito: Diminuição da excreção do ácido acetilsalić́lico

Interação medicamentosa potencial: Antipsicótico - IACh

Princípios ativos: Quetiapina/Donepezil

Efeito: Aparecimento de sintomas descontinuados como: efeitos extrapiramidais, rigidez generalizada

Interação medicamentosa potencial: Sulfoniluréia - Salicilato

Princípios ativos: Glibenclamida/Ácido acetilsalicílico

Efeito: Aumento do efeito hipoglicêmico

Interação medicamentosa potencial: Hipoglicemiante -

Hipotensor

Princípios ativos: Insulina /Losartan

Efeito: Aumento do efeito hipoglicêmico

Interação medicamentosa potencial: IECA - Diurético de alça

Princípios ativos: Captopril/Furosemida

Efeito: Aumento do volume urinário seguido de uma hiponatremia severa
Interação medicamentosa potencial: Beta bloqueador - IACh

Princípios ativos: Timolol/Donepezil,Atenolol/

Donepezil

Atenolol/Rivastigmina,Carvedilol/Donepezil

Efeito: Desenvolvimento de bradicardia intensa e hipotensão

Interação medicamentosa potencial: IECA - Salicilato Princípios ativos: Enalapril/ Ácido acetilsalicílico Captopril/ Ácido acetilsalicílico

Efeito: Redução do metabolismo dos ECA

Interação medicamentosa potencial: Inibidores da HMGCoA - Inibidores $\mathrm{H}^{+} / \mathrm{K}^{+}$ATPase

Princípios ativos: Sinvastatina/Omeprazol, Atorvastatina/Omeprazol

Efeito: Redução do metabolismo dos inibidores HMGCoA

Interação medicamentosa potencial: CYP2C9

substrato - Inibidores da isoenzima CYP2C9

Princípios ativos: Losartan/Omeprazol

Efeito: Redução do metabolismo dos substratos desta via.

Interação medicamentosa potencial: IECA -

Bloqueadores do canal de cálcio

Princípios ativos: Enalapril/Anlodipino

Efeito: Aumento do efeito hipotensor

Interação medicamentosa potencial: Insulina - Beta

bloqueador

Princípios ativos: Insulina /Atenolol

Efeito: Aumento do efeito hipoglicêmico

Interação medicamentosa potencial: Depressor SNC Depressor SNC

Princípios ativos: Quetiapina/Sertralina

Efeito: Aparecimento de sintomas como:

confusão, ataxia, fraqueza, sonolência e aumento do risco de depressão central

Interação medicamentosa potencial: Anticolinérgico - IACh

Princípios ativos: Dimenidrinato/Donepezil Efeito: Diminuição do efeito terapêutico do IACh

Interação medicamentosa potencial: IECA- Diurético poupador de $\mathrm{K}^{\dagger}$

Princípios ativos: Captopril/Espironolactona

Efeito: Aumento do volume urinário seguido de uma hipercalemia severa 
Ao avaliar nível de severidade das interações medicamentosas analisadas, observou-se que, $95,7 \%$ foram do tipo moderada e requerem o monitoramento da terapia farmacológica. Todavia, as interações entre os inibidores da enzima conversora de angiotensina (IECA) e bloqueador de canal cálcio (4,34\%) apresentaram nível de severidade menor e não requer que nenhuma mudança seja feita na terapia farmacológica (tabela 3).

Tabela 3- Distribuição dos pacientes submetidos à farmacoterapia das síndromes demenciais, segundo as interações medicamentos potenciais e respectivos níveis de severidade e riscos da terapia combinada. São Paulo-SP, 2010-2011.

\begin{tabular}{|c|c|c|c|c|}
\hline \multirow[t]{2}{*}{ Interação medicamentosa potencial } & \multicolumn{2}{|c|}{ Frequência } & \multirow[t]{2}{*}{ Risco } & \multirow[t]{2}{*}{ Severidade } \\
\hline & $\mathrm{n}$ & $\%$ & & \\
\hline Hipotensor - Hipotensor & 14 & 30,5 & $\mathrm{C}$ & Moderada \\
\hline Beta bloqueador - IACh & 4 & 8,8 & $\mathrm{C}$ & Moderada \\
\hline CYP3A4 substrato - Inibidores da isoenzima CYP3A4 & 3 & 6,5 & $\mathrm{C}$ & Moderada \\
\hline IECA - Salicilato & 3 & 6,5 & $\mathrm{C}$ & Moderada \\
\hline IECA - Diuréticos tiazídicos & 3 & 6,5 & $\mathrm{C}$ & Moderada \\
\hline Inibidores da HMGCoA - Inibidores $\mathrm{H}^{+} / \mathrm{K}^{+}$ATPase & 2 & 4,3 & $\mathrm{C}$ & Moderada \\
\hline Carvedilol - Inibidores isoenzima CYP2C9 & 2 & 4,3 & $\mathrm{C}$ & Moderada \\
\hline CYP2C9 substrato - Inibidores da isoenzima CYP2C9 & 2 & 4,3 & $\mathrm{C}$ & Moderada \\
\hline Salicilato - Diurético de alça & 2 & 4,3 & $\mathrm{C}$ & Moderada \\
\hline IECA - Bloqueadores do canal de cálcio & 2 & 4,3 & B & Menor \\
\hline Antipsicótico - IACh & 2 & 4,3 & $\mathrm{C}$ & Moderada \\
\hline Insulina - Beta bloqueador & 1 & 2,2 & $\mathrm{C}$ & Moderada \\
\hline Sulfoniluréia - Salicilato & 1 & 2,2 & $\mathrm{C}$ & Moderada \\
\hline Depressor SNC - Depressor SNC & 1 & 2,2 & $\mathrm{C}$ & Moderada \\
\hline Hipoglicemiante - Hipotensor & 1 & 2,2 & $\mathrm{C}$ & Moderada \\
\hline Anticolinérgico - IACh & 1 & 2,2 & $\mathrm{C}$ & Moderada \\
\hline IECA - Diurético de alça & 1 & 2,2 & $\mathrm{C}$ & Moderada \\
\hline IECA- Diurético poupador de $\mathrm{K}^{+}$ & 1 & 2,2 & C & Moderada \\
\hline Total & 46 & 100,0 & & \\
\hline
\end{tabular}




\section{DISCUSSÃO}

Os achados do presente estudo relativos aos aspectos clínico-demográficos da amostra foram similares aos de Scazufca et al. ${ }^{14}$ e Taylor et al. ${ }^{15}$ Scazufca et al. ${ }^{14}$ em estudo epidemiológico apontaram que a prevalência de síndromes demenciais no Brasil é de 5,1\% em idosos com idade superior a 70 anos, sendo que idosos na faixa etária de 65 a 74 anos se apresentam estáveis ao grau de demência. Em outro estudo, Taylor et al. ${ }^{15}$ relataram que a demência de Alzheimer afeta cerca de 20 milhões de pessoas no mundo. Dentre elas, 5\% estão acima de 65 anos, 40\% acima de 80 anos e $45 \%$ acima de 90 anos.

Todavia, a análise da demência de Alzheimer é de extrema valia, devido a sua alta prevalência entre os idosos, principalmente do sexo feminino, devido ao fato de contribuir para incapacidades psicomotoras nessa faixa etária, tornando esses idosos incapazes de cuidar da própria saúde, levando-os a serem dependentes de cuidadores e/ou responsáveis. ${ }^{2,15}$

No estudo de Taylor et al., ${ }^{15}$ foi descrita a prevalência de cuidadores do sexo feminino como $59 \%$, enquanto que os dados da presente pesquisa revelam um aumento do número de cuidadores do sexo feminino de $93,3 \%$, que por sua vez, assumem a responsabilidade mediante ao tratamento medicamentoso e/ou implementação do tratamento não farmacológico, sendo esses a maioria filhas ou esposas.

Os idosos com síndromes demenciais estão expostos a protocolos de tratamento prolongados, os quais requerem uma terapia de múltiplos fármacos. Embora não exista consenso sobre qual número expresse polifarmárcia, muitos autores a definem como a utilização de cinco ou mais medicamentos em associação ou como a administração de um medicamento do que clinicamente não necessita. A polifarmácia se configura como um dos problemas de segurança relacionado ao uso de medicamentos, porque é responsável pelo aumento do risco e da gravidade das reações adversas a medicamentos e pode precipitar interação medicamentosa, causar toxicidade cumulativa, ocasionar erros de medicação, reduzir a adesão ao tratamento e elevar a morbi-mortalidade. ${ }^{8,16,17}$

Contudo, o número de medicamentos prescritos por paciente tem gerado desconforto por parte dos pacientes e/ou responsáveis por falta de disponibilidade e orientação do cuidador em estar junto com o idoso no horário de administrar os medicamentos, ou ainda porque o paciente se nega a tomar os medicamentos, levando-o a não aderir ao tratamento farmacológico. A não adesão ocorre, portanto, quando o comportamento do paciente e/ou responsável pela administração da terapia farmacológica não coincidem com as recomendações orientadas. ${ }^{18}$

As interações medicamentosas têm sido um importante objeto de investigação, uma vez que o potencial para interação medicamentosa aumenta com o avançar da idade, o qual se justifica com a mudança do perfil farmacológico decorrente do processo de envelhecimento. ${ }^{19,20}$

É evidente que a falta de estudos na literatura sobre as interações medicamentosas, em especial para os idosos portadores de síndromes demenciais, tem gerado uma relevância clínica significativa, por parte dos profissionais de saúde, no que se refere aos problemas relacionados ao uso de medicamentos, levando a uma carência de orientação aos cuidadores, quanto a doença e tratamento medicamentoso, bem como aos possíveis efeitos relacionados às interações medicamentosas potenciais mencionadas na amostra, razão esta pela qual o estudo apresenta limitações quanto à avaliação do comprometimento cognitivo dos pacientes, tornando-se de fundamental importância a participação interdisciplinar dos profissionais de saúde.

\section{CONCLUSÃO}

Apesar de os pacientes portadores de síndromes demenciais submetidos ao tratamento medicamentoso se apresentarem estáveis, no que 
diz respeito à avaliação do comprometimento cognitivo-demencial, a interação medicamentosa, como evento precursor do uso de medicamentos, se torna um parâmetro importante no que se refere à evolução clínica do paciente, podendo resultar em efeitos potencialmente perigosos, bem como quando associados podem melhorar ou intensificar o efeito farmacológico esperado, como o visto nas interações medicamentosas mediadas ao uso de medicamentos hipotensores, havendo relação indireta com o grau de demência e consequentemente, evolução clínica dos pacientes avaliados pela escala de Clinical Dementia Rating (CDR).

\section{REFERÊNCIAS}

1. Kusano LTE. Prevalência da polifarmácia em idosos com demência [dissertação de mestrado]. Brasília: Faculdade de medicina da Universidade de Brasília; 2009.

2. Carvalho MFC. A polifarmácia em idosos do município de São Paulo - Estudo SABE - Saúde, Bem-estar e Envelhecimento [dissertação de mestrado]. São Paulo: Faculdade de Saúde Pública da Universidade de São Paulo; 2007.

3. Lebrão ML, Duarte, YAO. SABE - Saúde, Bem-estar e Envelhecimento - O Projeto Sabe no município de São Paulo: uma abordagem inicial. Brasília: Organização Pan-Americana da Saúde 2003; 255 p.

4. Lyra Júnior DP, Amaral RT, Veiga EV, Cárnio EC, Nogueira MS, Pelá IR. A farmacoterapia no idoso: revisão sobre a abordagem multiprofissional no controle da hipertensão arterial sistêmica. Rev LatinoAm Enfermagem 2006;14(3):435-41.

5. Locatelli J. Interações medicamentosas em pacientes hospitalizados. Einstein (São Paulo) 2007;5(4):343-6.

6. Nies AS, Spielberg SP. Princípios da terapêutica. In: Hardman JG, Limbird LE, Molinoff PB, Ruddon RW, Gilman AG, editores. Goodman \& Gilman: as bases farmacológicas da terapêutica. 9. ed. New York: McGraw-Hill; 1996. p. 31-44.

7. Guastaldi RBF. Interações medicamentosas potenciais: um estudo dos antimicrobianas utilizados em pacientes submetidos ao transplante de medula óssea [dissertação]. São Paulo: Escola de enfermagem da USP; 2006.
Nesse contexto, as síndromes demenciais figuram como importante problema de saúde pública, devido aos custos onerosos com medicamentos e internações, além de provocar danos emocionais, físicos, sociais e financeiros para os pacientes, familiares e cuidadores.

\section{AGRADECIMENTOS}

Agradecemos ao Instituto Paulista de Geriatria e Gerontologia José Ermírio de Moraes, pelo acesso e disponibilidade junto às equipes da farmácia e multiprofissional, permitindo assim a realização desta pesquisa.

8. Cipolle RJ, Strand LM, Morley PC. O Exercício do Cuidado Farmacêutico. Brasília: Conselho Federal de Farmácia; 2001

9. Grupo de Investigación en Atención Farmacéutica; Universidad de Granada; Grupo de Investigación en Farmacología; Universidad de Granada; Fundación Phamaceutical Care España; Sociedad Española de Farmacia Comunitaria. Tercer consenso de Granada sobre problemas relacionados con medicamentos (PRM) y resultados negativos asociados a la medicación (RNM). Ars Pharm 2007;48(1):5-17.

10. Loyola Filho AI, Uchoa E, Firmo JOA, LimaCosta MF. Influência da renda na associação entre disfunção cognitiva e polifarmácia: Projeto Bambuí. Rev Saúde Pública 2008;42(1):89-99.

11. Doença de Alzheimer [Internet]. Brasília: Ministério da saúde; 2002 [acesso em 16 ago 2010]; Protocolo clínico e Diretrizes terapêuticas - Medicamentos Excepcionais; [aproximadamente 2 p.]. Disponível em: http://dtr2001.saude.gov.br/sas/dsra/ protocolos/do_d09_00.htm

12. World Health Organization. The Anatomical Therapeutical Chemical: Classification System with Defined Daily Doses (ATC/DDD) [homepage na Internet]. Geneva: WHO; c2007. [atualizado em 16 dez 2006; acesso em 16 mar 2007]. [aproximadamente 2 telas]. Disponível em: http://www.who.int/ classifications/atcddd/en/.

13. Conceição LFS. Saúde do idoso: orientações ao cuidador do idoso acamado. Rev Med Minas Gerais 2010;20(1):81-91. 
14. Scazufca M, Menezes PR, Vallada HP, Crepaldi AL, Pastor-Valero M, Coutinho LM, et al. High prevalence of dementia among old adults from poor socioeconomic backgrounds in São Paulo, Brazil. Int Psychogeriatr 2008;20(2):394-405.

15. Taylor LO, Dellaroza MSG. A realidade da atenção a idosos portadores da doença de Alzheimer: uma análise a partir de idosos atendidos em serviços públicos. Semina: Ciências Biológicas da Saúde 2010;31(1):71-82.

16. Pinho FL. Demência: a marcha diagnóstica no âmbito dos cuidados de saúde primários [dissertação de mestrado]. Covilhã, Portugal: Universidade da Beira interior; 2007.
17. Steinman MA, Rosenthal GE, Landefeld CS, Bertenthal D, Sen S, Kaboli PJ. Conflicts and concordance between measures of medication prescribing quality. Med Care 2007;45(1):95-9.

18. Dewulf NLS, Monteiro RA, Passos ADC, Vieira EM, Troncon LEA. Adesão ao tratamento medicamentoso de pacientes com doenças inflamatórias intestinais acompanhados no ambulatório de um hospital universitário. Arq Gastroenterol 2007;44(4):289-96.

19. Secoli SR. Polifarmácia: interações e reações adversas no uso de medicamentos por idosos. Rev Bras Enferm 2010;63(1):136-40.

20. Brunton LL, Lazo JS, Parker KL, editores. Goodman \& Gilman: as bases farmacológicas da terapêutica. 11. ed. Rio de Janeiro: Mc Graw-Hill; 1996. 